\title{
Categorial Occasionality and Transformation: Analyzing Culture in Action
}

\author{
Sally Hester $\cdot$ Stephen Hester
}

(C) Springer Science+Business Media B.V. 2012

\begin{abstract}
Our focus in this article is on some uses of categorial transformations. The discussion is divided into two main parts. In the first part, we begin by outlining our approach, namely membership categorization analysis (MCA), indicating the origins of the term and elaborating the conception of MCA as an 'occasioned' members' apparatus. We then explain what we mean by the concept of categorial transformation, review some of the very few previous studies which have investigated this phenomenon and which are pertinent to its further study, and indicate how categorial transformation serves to embody and illustrate the occasionality of MCA. In the second part of the article, we present an analysis of two extracts from a transcript of the naturally occurring talk-in-interaction which occurred in a particular family meal, involving two children and their parents. A variety of categorial transformation practices in this data are identified and their particular local usages described. In the concluding discussion we consider our argument and our analysis in light of previous discussions of the trajectory of Sacks' work.
\end{abstract}

Keywords Ethnomethodology - Membership categorization analysis · Occasionality · Categorial transformation · Culture-in-action

\section{Introduction}

Our focus in this article is on some uses of categorial transformations. The discussion is divided into two main parts. In the first part, we begin by outlining our approach, namely membership categorization analysis (MCA), indicating the origins of the term and elaborating the conception of MCA as an 'occasioned' members' apparatus. We then explain what we mean by the concept of categorial

\footnotetext{
S. Hester $\cdot$ S. Hester $(\bowtie)$

School of Social Sciences, Bangor University, Bangor LL57 2DG, UK

e-mail: sos032@bangor.AC.UK
} 
transformation, review some of the very few previous studies which have investigated this phenomenon and which are pertinent to its further study, and indicate how categorial transformation serves to embody and illustrate the occasionality of MCA. In the second part of the article, we present an analysis of two extracts from a transcript of the naturally occurring talk-in-interaction which occurred in a particular family meal, involving two children and their parents. A variety of categorial transformation practices in this data are identified and their particular local usages described.

\section{Membership Categorization Analysis}

Since its origins in the work of Sacks (1992a, b), membership categorization analysis (MCA) has developed into a distinctive genre of ethnomethodological inquiry along with its sequential counterpart in conversation analysis. Sacks, of course, did not distinguish as a separate field of inquiry membership categorization analysis (MCA) from conversation analysis (CA) and there remain in place strong arguments (some of which are touched on below) for the maintenance of robust links between the categorial and sequential 'dimensions' of social life. Nevertheless, the distinctiveness of these dimensions has been sufficient to provide for their institutionalization as discrete domains of inquiry in their own right.

The term 'membership categorization analysis,' was proposed firstly by Eglin and Hester (1992) as a replacement for 'MCD analysis' (the term under whose rubric the distinctive categorial dimension of social life had been analyzed during the 1970's and 1980's). ${ }^{1}$ The reasons for this proposal were not merely aesthetic, nor were they name-changing for its own sake (cf. Schegloff 2007a), they were that 'MCD analysis' privileged the analysis of membership categorization devices, and whilst this privileging acknowledged the originality of Sacks' notion of category collections, it obscured the fact that whilst membership categories always belong to some collection and whilst their intelligibility for members depends crucially on their membership in a collection, it is also equally the case that category collections are dependent for their intelligibility for members upon which categories they collect together. If neither collections nor categories are intelligible without the other, then a term which recognized this fact seemed appropriate to say the least, hence the term 'membership categorization analysis' as a term which covered the full range of categorization practices without giving priority to any practice in particular.

There are now numerous introductions which discuss the 'apparatus' or 'machinery' consisting of the various components used in categorization practices, and so these will not be repeated here. ${ }^{2}$ Such discussions emphasize the selectivity of membership categorizations. Like descriptions generally (see Heritage 1984), membership categorizations are always selections from alternatives, a point made

\footnotetext{
1 For a review of the work conducted under the auspices of 'MCD analysis,' see Eglin and Hester (1992).

2 See, for example, Eglin and Hester (1992), Hester and Eglin (1997a), Coulter (1991), Benson and Hughes (1983) and Francis and Hester (2004).
} 
most forcibly by Sacks in connection with Pn-adequate devices, and a major theme in MCA from Sacks onwards has been to explore the methodical ways in which various 'considerations' are taken into account by members in selecting categorizations on particular occasions. In view of the widespread appreciation of categorial selection, we will not pursue the topic further at this juncture. Instead, before considering the concept of categorial transformation, we wish to focus attention on the occasionality or situatedness of members' MCA.

With respect to the occasional or situated character of membership categorization practices, it was emphasized by Hester (1994) and Hester and Eglin (1997a, b) that MCA takes a particular stance toward 'culture' (see also Watson 1997; Baker 2000). The 'machinery' of MCA — category collections, membership categories, category predicates, etc. - can be thought of as one aspect of a society's culture but in the sense of 'culture-in-action' or as improvisational cultural practices rather than as a body of decontextualized knowledge, practice and convention. One way to appreciate the occasionality of the machinery is to recognize the 'branching texture' of collections and categories. A category can become a collection, and vice versa. Categories may belong in collections but they may then also be collections themselves, that is, provide for a new 'branch' of categories. It is important to recognize that this is not just an abstract formulation of the apparatus. Whether something is being used this time as a category or a collection is an empirical matter. Furthermore, it may well be that certain consequential matters and actions turn on whether it is being used in one way or the other, that is, as either a category or a collection.

However, MCA is an occasioned or situated activity in a much deeper sense than this. In this regard, it is useful to recall that Sacks (1992a: April 17th 1968) drew a distinction between 'occasioned' and 'natural' collections of categories in one of his discussions of the phenomenon of 'topic'. The distinction is easily misunderstood if it is taken to imply that some collections are 'occasioned' whilst others, that is, 'natural' collections, are not. Thus, Sacks said that an 'occasioned' collection is one assembled on the occasion of some topic having been introduced in a conversation and, as a result, some 'strange bedfellows' may be collected together for the duration of the topic. The example he uses is the consecutive mentioning of 'child, fourteen' and 'dog,' two membership categories with respect to which a collection is not immediately transparent. However, once it is known that the parties to the conversation are talking about 'potential obstacles to renting an apartment' then it is reasonably the case that these membership categories could both be included in such a collection. The relevance of their mentioning would have been provided for, that is to say 'occasioned,' by the topic at hand. Sacks also said that a 'natural' collection was one where if the name of the collection was known, then a member of the culture could name its members. This might be mistakenly understood as meaning that 'natural' collections are part of a decontextualized cultural apparatus, a 'stable' cultural framework that the analyst can use to make sense of (or impose sense upon) data. Such 'culturalism' obscures rather than illuminates members' membership categorization analytic practices. Sacks recognized clearly that the collection to which a category belongs is an occasioned matter, and this applied to both 'occasioned' and 'natural' collections. In a discussion of category bound activities 
(Sacks 1992a: Spring 1967, Lectures 12 and 13) he indicated that the collection to which a category belongs will depend on the meaning of the category on the specific occasion of its use. He used as an example the category 'baby,' making it plain that it may belong to a number of collections, including not only 'family' and 'stage of life device' but also 'boyfriend/girlfriend' and 'married couple'. By extension, then, not only may the category 'baby' have a variety of different activities predicated of it- 'crying' is an activity bound to the category 'baby' only on the assumption that the baby in question is an infant (Sacks 1992a: Spring 1967, Lecture 13: 584)—but also collections can mean different things and therefore be composed of different categories. In short, all collections, whether 'natural' or 'topically occasioned'-are assembled on particular occasions (Hester and Eglin 1997a, b; Watson 1997).

Accordingly, as an aspect of culture, the machinery of membership categorization analysis is conceived as a situated and occasioned machinery. This means not simply that the machinery is put to localized uses but that the machinery itself is locally assembled on the occasions of its use (Watson 1997). This does not mean that collections, categories and their predicates are invented each time they are used; it means that collections, categories and predicates are always 'indexical expressions,' and irremediably so (Garfinkel 1967). The sense or meaning of a collection, a category or a predicate will always be relative to, and require recourse to, a particular local social context as accomplished and oriented to by members; it will depend upon how the collection-name, category or predicate is being used on a particular occasion.

'Culture,' then, is to be found in action and categories are always 'categories in context' and this means that the task for MCA is to discover how collections, categories and predicates are used on the occasions of their occurrence rather than presuming their stable cultural meanings. With its origins in ethnomethodology and Sacks' conversation analysis, MCA is guided in this task by a number of methodological principles. It is beyond the scope of this article to provide a thorough review of these (Sacks 1984; Schegloff 1993; Heritage 1984; Francis and Hester 2004). However, in terms of the present discussion it is important to stress that membership categorization analysis refers not only to and not primarily to the analysis of members' categorization practices but also and most emphatically to the naturally occurring membership categorization analyses in members' practices. This does not preclude the self-reflective analysis of the analyst-as-a-member's practices but it does preclude the making of mere categorial observations by an-analyst-asmember. ${ }^{3}$ Sacks (1992a: Spring 1967, Lecture 12: 583) makes it very clear that this 'is not yet anywhere near good enough' in his discussion of category bound activities. Any such observations can only be a starting point for analysis, not its conclusion. Furthermore, it is arguably the case that the main strength of 'selfreflective' MCA is pedagogical; it serves as a useful device for explicating the concepts and methods of doing MCA but it falls short of demonstrating (other than via a presumption of shared 'culture') that anyone other than the analyst actually used the membership categorization machinery in question. An adequate

\footnotetext{
3 The 'model' for self-reflective MCA is Sacks (1974), used in, for example, Payne (1976), Lee (1984), Eglin and Hester (1992), Hester and Eglin (1997a), Francis and Hester (2004).
} 
demonstration requires that the analyst's claims about members' membership categorization practices are made subject to a principle of empirical authorization or demonstrable relevance in the details of naturally occurring talk-in-interaction. In order to achieve such an empirically authorized account, the analyst is required to ascertain which categorizations are demonstrably relevant for and used by the participants in the talk-in-interaction under consideration. This is achievable not by invoking what is 'hearable' or 'recognizable' from the analyst's point of view but by showing, from the details of the talk-in-interaction, how the participants themselves analyze each other's categorization (and other) practices.

\section{Occasionality and Categorial Transformation}

An appreciation of the occasionality and assembled character of the machinery of membership categorization analysis is afforded especially in instances of categorial transformation. As indicated above, the point of saying that the machinery is occasioned is that (a) whether something is a category or a collection, (b) what collection a category belongs to and (c) what a collection consists of, is a matter for empirical investigation, discoverable in the specifics of talk-in-interaction. The apparatus for doing membership categorization analysis for various practical purposes is revealed in the course of the analyses that members do, and not to be presumed in advance of such empirical instances. Culture is to be discovered in action, not assumed a priori and then used as an analyst's resource. It is in their talkin-interaction (and other social actions) that categories of persons and objects are collected, categories given different meanings, collections given different categories; it is there that collections can become categories and vice versa, collections being assigned different categories, the same categories being allocated to different collections, and so forth. The apparatus, in other words, is built locally and situationally for particular practical purposes. Categorial transformations are occasions of the use of the apparatus of MCA which exhibit this occasionality.

As was indicated in Hester and Eglin (1997a, b: 3), categorizations may be accomplished not only with respect to persons (the original reference for 'membership categories') but they may also be used in respect of collectivities (Coulter 1982; Jayyusi 1984; Sharrock 1974) and non-personal objects of various kinds (McHoul and Watson 1984), including what have traditionally been referred to in conversation analysis as 'conversational objects' or 'sequential objects' (for example, questions, answers, story-invitations, requests, instructions, etc.). Indeed, that persons are able to distinguish one type of conversational object from another suggests some course of membership categorization analysis. The ways in which the use of these categorizations of collectivities and non-personal objects display organizational features similar to those of personal membership categories remains a matter for empirical investigation (Hester and Eglin 1997a, b: 3).

One property that they appear to have in common is their transformability. Just as persons may transform or have transformed their membership categories, so also may categories of non-personal object be so transformed (and we suggest collectivity categorizations as well, though our analysis in this article does not 
address this particular issue). This transformability of meaning in the course of social interaction is a property which has long since been appreciated, for example in discussions of the properties of indexical expressions (Garfinkel 1967), of the flexibilities of natural language use (Heritage 1978), of the situated occasionality of membership categories and category collections (Sacks 1992a; Hester and Eglin 1997a, b), of the 'double duty' character of 'sequential' objects (Schegloff 2007a) and of 'transformative answers' in resistance to questions (Stivers and Hayashi 2010). ${ }^{4}$ However, to our knowledge, transformation as a categorial conversational activity has seldom been investigated as a practical matter in conversational interaction in its own right. Several previous studies within the ethnomethodological and conversation analytic literature indicate the potential of such a research focus.

Two suggestive examples of such research can be found in Sacks (1992a, b). The first concerns the 'ambiguity of warnings,' and relates directly to what was mentioned above about categories of conversational object. Thus, as Sacks (1992a: Spring 1967, Lecture 13: 586) indicates:

...there's a wonderful kind of ambiguity involved in receiving warnings. That is to say, what one who receives an observable 'warning' can make of it, involves that observable 'warning' in having alternative possibilities. The big thing is that a 'warning' can be heard as a 'challenge'. In fact their linguistic forms, for example are often quite indistinguishable. Now, that relation of 'warning/challenge' can turn on the categorial positions of the warned possibly challenged - person. Specifically, if a 'boy' is warned qua 'boy,' and turns that warning into a challenge, then he takes the, now, 'challenge' as a chance to prove that he is not in the first instance - or is no longer - a 'boy'.

Two kinds of categorial transformation are indicated here. Firstly, there is the categorial transformation of the conversational object of 'warning' into a 'challenge'. Second, there is the transformation (or at least possible transformation), made possible by the transformation of warning into challenge, of the category membership of one who is a 'boy' into one who is no longer to be identified in this way.

The second example from Sacks is to be found in his analysis of 'requests, offers and threats' and 'the 'old man' as an evolved natural object' (Sacks 1992b: Winter 1971, March 11: 318-331). In this analysis, Sacks shows, on the one hand, how categories of conversational object, and on the other hand, membership categories (of persons) are transformed in the course of some mealtime talk initiated by an offer of some food (herring) to Max, a recently widowed 'old man'. The sequence begins with a request by Ethel, who is Max's stepdaughter-in-law, to Ben who is Max's stepson-in-law, to bring out some of the herring so that Max can try it. Max declines this offer whereupon attempts are made to persuade him to change his mind. Several transformations are involved in this sequence. Firstly, as Sacks (1992b: 327) puts it, "we get a movement from a variety of offers, to a request—not to eat but to taste, "Max, one piece," through the warning format, "You better eat something because you're gonna be hungry before we get there," to, finally, some

\footnotetext{
${ }^{4}$ See also Buckner (1970) for a classic 'phenomenological' account of transformation practices.
} 
sort of quasi-threat, "I don't want you to get sick"'. Secondly, the herring, as a category of food, itself 'becomes transformed to "Eat something"' (any category of food) (Sacks 1992b: 327). Thirdly, there occur some concomitant transformations in the category memberships or 'operative identities' of the parties involved. As Sacks (1992b: 327-328) indicates, 'the sequence of offer-transforms operates via a series of identity changes that progressively provide further transforms'. Specifically, the identity changes involve Max becoming progressively transformed into a 'stubborn old man' because of his rejections and Ben and Ethel into persons who are now burdened with the responsibility of taking care of him. As Sacks (1992b: 330) indicates:

As they proceed through the sequence and Max keeps doing the rejections, that status of his - that he has no one to take care of him - can be made more alive. Their reoffers can be specifically turning him into a 'stubborn old man'. Seeing the stubborn old man, they can see that he's the stubborn old man that they're responsible for, i.e. that he doesn't take care of himself. In part, then, the person he becomes in the sequence, the person they have got to take care of, is an identity that the sequence brings into focus.

Jayyusi (1984) discusses how the sense of an event can be transformed if the membership category of the person involved is changed. She uses as an example, taken from the transcripts of the Scarman Tribunal, of some questioning of a witness in which the Chairman of the Tribunal selects 'old men with heart attacks' and 'young men with bloody noses' as the age groups of people present in the events being discussed, thereby excluding women and children as pertinent categories of participant. The lawyer's next question achieves a further transformation by selecting only 'young men' as the category of person who had sustained injuries caused by violence. As Jayyusi (1984: 106) points out, this category transformation serves to shape the sense of what occurred in the events being examined. That is, the category 'young man' permits inferences that, say, 'old man' or 'young woman' do not. If the person with the bloody nose is a 'young man,' then it may be supposed that he may well have had a hand in his own injury, through participating in a fight perhaps. Contrastively, had the category selected been a 'young woman,' then it may be supposed that she was a victim of some kind, perhaps of domestic violence. These common-sense inferences about the relationship between injuries caused by violence and the category membership of the person suffering it comprise aspects of a cultural 'grammar' (Eglin and Hester 1992) for understanding events and reports of them. As Jayyusi's analysis demonstrates, such transformations can have significant procedural consequences in contexts such as tribunals and trials.

Sharrock and Turner (1978) also discuss categorial transformation. Their data consists of calls to the police, a 'conversational environment for equivocality' in so far as callers' complaints may be interpreted either 'literally' (where they are understood in terms of "what the speaker "appears to say") or 'in some way [that] undercuts the literal version' (Sharrock and Turner 1978: 186). In particular, Sharrock and Turner discovered an orientation on the part of callers which concerned possible transformations of their calls from bona fide to maliciously motivated and of themselves from 'genuine' callers into 'malicious' callers. Such 
transformation can occur especially when those who are 'complained against' are identified, either categorially or by name or both. Such identifications can imply, for the police in this instance, corresponding categorial incumbencies for the complainant which serve to undercut the complaint. It is therefore unsurprising that callers may design their complaints 'just in order to recognize and negate the possibly equivocal involvement of neighbours in the construction of a complaint' (Sharrock and Turner 1978: 188). As the authors (1978: 187) put it:

One of the fates of stories, narratives, and anecdotes is that their recipients may perform transforms on them, either in later retellings or "interpretively," that is, in figuring out for themselves the sense of what they have been told .... Recipient can recast the part teller assigns himself in his telling, with the result that the whole narrative undergoes a shift so as to "tell a different story"; and an assessment that the remarks are equivocal can motivate recipient to operate the transform. Thus, "complaints" can undergo such a shift, so as to yield a story now focused on complainant, and complained-againsts can correspondingly appear in this version as victims.

Clearly, the transformability of conversational objects and the category membership of conversationists is not limited to calls to the police and, as Sharrock and Turner (1978: 1990 suggest, provide 'analytical topics' for further research.

Finally, Eglin and Hester (2003) analyze how the selection of different categorial resources serves to constitute a series of different news stories about 'the Montreal Massacre'. The initial news story is of a shooting in a college and the membership categories are 'gunman,' 'victims,' 'police,' and other possible 'suspects'. As the newspaper coverage of the event unfolded, however, a number of other stories were told and the transformation of the membership categories of the parties to the event was a key component of the methodology of these stories. For example, for the 'horror' story, the 'victims' became 'people screaming, shouting and yelling' and 'scores of horrified students streamed' from the 'terrifying scene' and for the story of 'tragedy,' the categorial transformations of the victims were to 'young people' and 'students' (at the start of their lives), 'daughters,' 'sisters,' and 'friends' (their tragic loss to their families and friends) and 'mourners waiting in silence to pay final respects'.

As each of these studies suggests, categorial transformations can be accomplished in a variety of ways and may be used in the achievement of different kinds of social action. We will now turn to some transformations in our data.

\section{Setting and Data}

The setting in which our research on transformation was conducted is that of a family meal, a dinner to be more precise, which occurred at the end of a weekday during which the two parents (Jen and Harry) present had been out to work and their two children, a boy (Russell) aged 13 and a girl (Maggie) aged 7, had been attending school. The dinner takes place in the kitchen where the participants are seated around a kitchen table. The meal is begun when the food is cooked and ready to be brought to the table and served. At this time, Harry, the father, has not yet returned 
home, although he has telephoned to say he is on his way. He arrives some 20 minutes later, which is about halfway through the meal. The entirety of the talkin-interaction from when the mother announces that dinner is ready, asking her children if they have washed their hands and summoning them to the table was audio-tape recorded with the permission of the parents and children. This tape recording was then transcribed according to the transcription conventions of conversation analysis (Jefferson 1978).

Our discovery of some categorial transformations in these materials was 'unmotivated'. We did not set out to investigate such a phenomenon, we just happened to come across them when we were looking closely at our data. Having discovered a first transformation, and then a second, we were then motivated to see if we could assemble a collection of them. Our collection is not large, since we have limited ourselves to those transformations that we discovered in the data that we had to hand. In what follows, then, we will analyze two data extracts which contain five instances of categorial transformation. With respect to the first extract we analyze an instance of a transformation of a category of a non-personal object, namely a 'normal potato,' from a category of potato into a collection of potatoes during the course of the serving of food in the meal. The second extract contains four transformations: a collection is transformed into a category, a category of conversational object is transformed, a category is transformed into a collection and the collection to which an object belongs is transformed. These transformations are not accomplished for their own sake but, as we will show, they are involved in the achievement of various actions, including serving the food, avoiding a potential outbreak of conflict, furthering the progressivity of a conversation and making a joke. Each of these transformations is categorial in character and all of them illustrate the occasionality of the MCA apparatus and that such transformations are done on particular occasions for specific practical purposes. That is to say, whilst the MCA apparatus is inherently occasional and indexical, it is also occasioned in the sense of being shaped and built in relation to specific and situated actions.

\section{Analysis 1}

At the beginning of the meal, after the children have sat down at the kitchen table, Jen (their mother) serves them with various items of food. This takes several minutes as the items are brought from the stove, one at a time, to the table. Jen offers these items to the children, again one at a time. Potatoes are the first item to be offered, as the following extract (1) indicates:

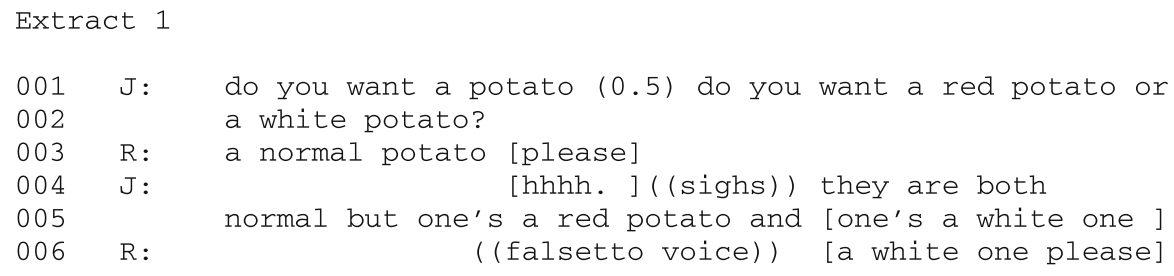


The occasionality of the MCA apparatus, and specifically the occasionality of categories and collections is well illustrated in this extract. As can be seen, Jen's offer of potatoes is constructed as a question which inquires as to which of two categories of potato her son, Russell, would like. This offer presents a choice to be made between two candidate categories of potato, so that a selection of one or other of these candidates would constitute an appropriate method for completing the adjacency pair, and responding to the offer contained in the question. In other words, the offer is designed for an acceptance of one of the categories of potato that are on offer: does he "want" a 'red' or a 'white' potato, where the projected answer is one which makes a choice between the two. However, as his utterance at line 003 makes evident, Russell does not choose one of the two categories of potato on offer. Instead, he names a different category of potato that he would accept, namely a 'normal potato'. This can be heard as an alternative category to the two categories selected by Jen in her offer. Given the adjacency pair organization of the projected sequence-offer followed by either acceptance (of one category or the other) or declination-his selection of 'a normal potato' can be understood as a declination of the choice of potatoes on offer. However, he is not declining an offer of potato as such, he is declining the choice of potato he has been offered. He will have a potato but he will accept it on his own terms, not on the terms provided by his mother. His selection, then, of a 'normal potato' can be understood as an alternative category to the two categories of potato offered by his mother.

Russell's alternative category of the potato which he will accept could be interpreted in various ways, perhaps as a kind of challenge, a 'wind-up,' a provocation or as play, to name just a few possibilities. However, what is more important than these conjectures is the nature of Jen's response to it. Thus, her initial response (at line 004) is a sigh: 'hhhh.,' which is recognizably exasperated in tone and, as such, can be heard to accomplish some action. One use of sighs is their conveyance of assessments of the just-completed utterances or actions, or indeed current courses of action. In particular, sighs are often positioned after delivery of some news or statement that places a burden of some sort on its recipient, a task that now has to be dealt with, and prior to the task's address. In this case, Jen's sigh can be understood as indicating (possibly complaining) that Russell has just now presented her with a task that she will have to reluctantly perform. Some parents may sigh prior to an admonishment or censure, but here Jen's sigh is positioned prior to an explanation; it is this task that she now has to do, and accordingly does. She explains that both categories of potato she has offered him are 'normal'.

\section{Transforming a Category into a Collection}

The key point, however, is that if her explanation is inspected closely it can be seen that it involves a transformation of 'normal potato' from an alternative category of potato into a collection of categories of potato. What is important here is that Jen's reply that 'they are both normal but one is a red one and one is a white one' spells out the apparatus, that is the particular configuration of collection and categories to be used on this occasion. Thus, she does so by transforming his selected alternative 
category of potato into a collection; she replaces his alternative category with an inclusive collection, one which includes both red and white potatoes. As she says, 'they're both normal'.

Whilst the data shows Jen's categorial transformation of a category into a collection, and thus demonstrates the occasionality of the apparatus of MCA, what also needs to be said is what it is that occasions the use of such a transformation. We suggest that it is accomplished in order to 'progress' the serving of the food. Russell's initial rejection of her offer of potatoes can be understood, on the basis of Jen's response to it, as a disruption of that task. Her transformation therefore deals with the potential diversion from completion of the task. It re-establishes progressivity of the sequence initiated by her first offer.

As the data shows, Russell makes no further issue in connection with how to describe potatoes: he chooses a white one. The categorial wind has been taken out of Russell's disruptive sails. His choice of an alternative category disrupted the progressivity of the sequence projected by Jen's offer. Her incorporation of her categories into a collection bearing the name of his selected alternate category serves to remove the source of disruption. Since they are both talking about 'normal potatoes,' there is no cause for further diversion from the task at hand. As the data shows, once Jen has said that 'they are both normal,' the 'insertion sequence' that began with his declination and conditional acceptance of Jo's offer is completed and Russell then produces a preferred response to Jen's original offer: he will have a white one.

\section{Analysis 2}

Our second and longer extract from the data contains four transformations. The extract is taken from that part of the meal where Harry, the father, has just returned home and has now joined the others at the table. After some talk between Harry and Jen about their respective day's work, Harry turns to his son and asks him about his day at school. The data is as follows.

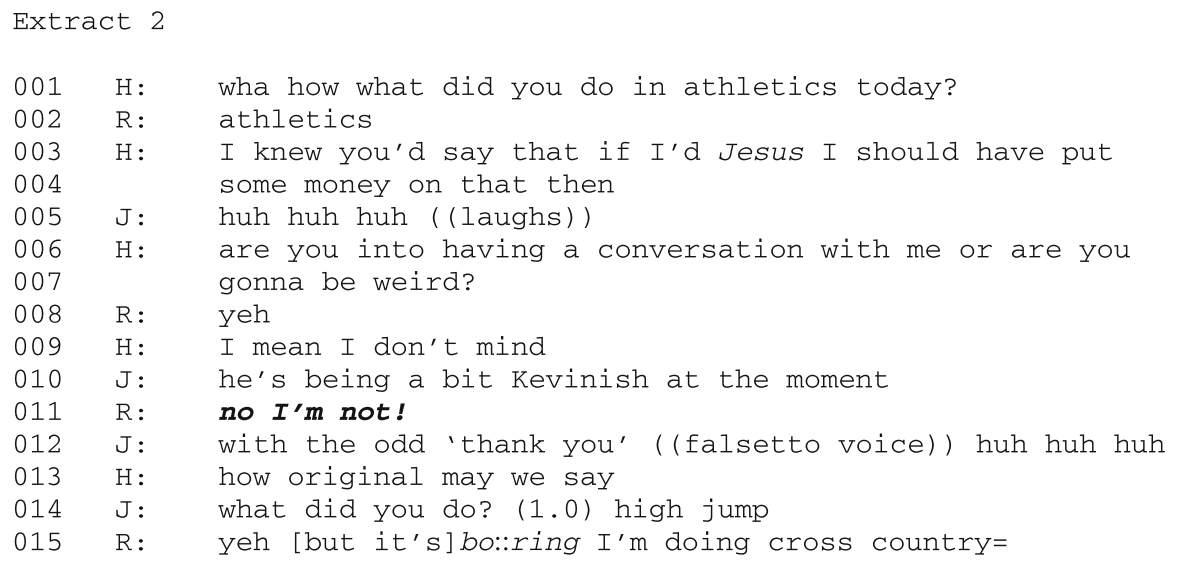




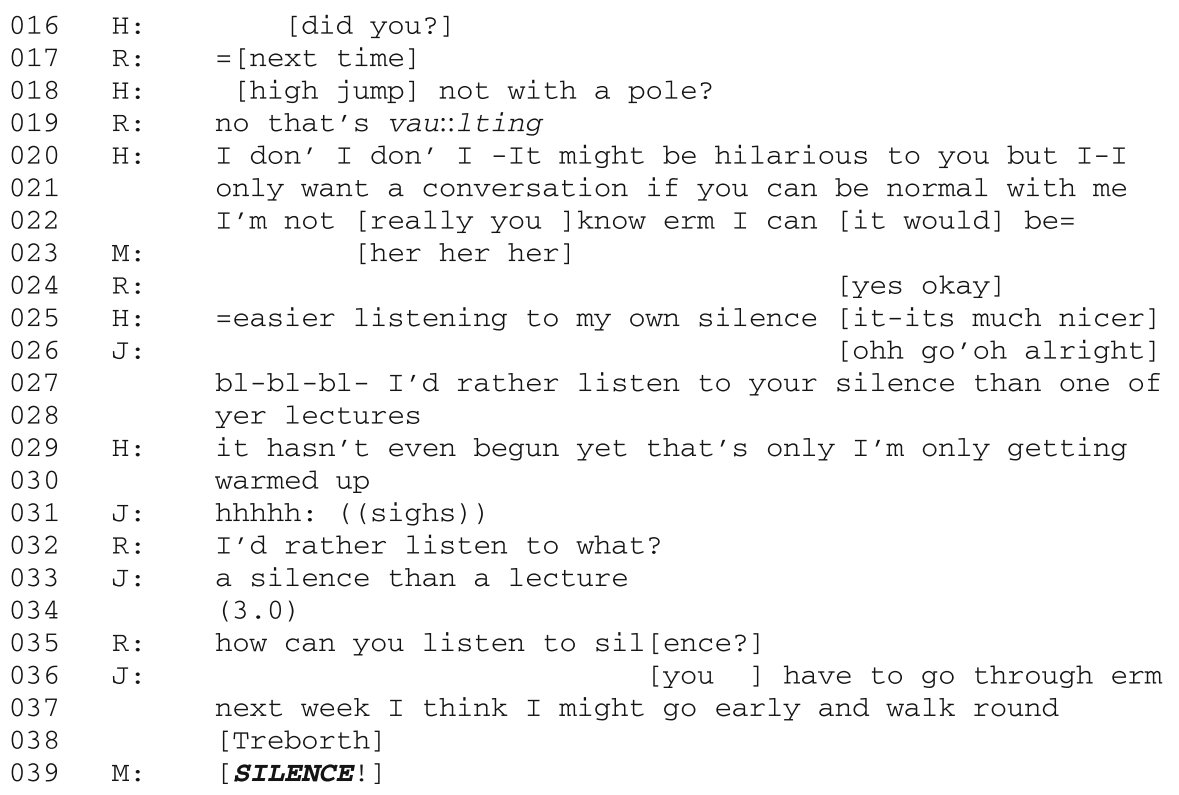

The first two lines contain two transformations. The first of these involves a transformation of a collection into a category, the second consists of a transformation of a category of conversational object into a different category of conversational object. The first is the vehicle for the achievement of the second. We will consider each of these transformations and then turn to the MCA that the parties to this talk-in-interaction do in relation to them.

\section{Transforming a Collection into a Category}

With respect to the transformation of a collection into a category, it can be seen that Harry asks Russell 'what' he did 'in athletics today'. Clearly, the question (or more precisely, story-invitation - see below) can be understood to provide a collection (or class) of topically related items which may be addressed by Russell. In asking what Russell did in athletics, 'athletics' can be understood to be something inclusive, as naming a located (at school) collection of things (activities in this case) which constitute them. The collection 'athletics' subsumes a range of activities, such as running, sprinting, jumping, throwing, etc. each of which can operate as collections themselves composed of further sub-categories of athletics. However, Russell declines to treat athletics as a collection for elaboration. In other words, Russell transforms a category collection into a single category. As far as Russell is concerned, there is therefore no collection whose constituents require itemization or elaboration as 'the day's athletics'. Rather, there is only the single category of activity, namely athletics. The various 'whats' projected by Harry's collection, have been transformed into the single 'what' of Russell's category. Had Russell 
mentioned a category of athletics, then this would have been a standard topical development of the conversation (Sacks 1992a: Lecture April 17, 1968) However, the repetition of the name of the collection as an answer takes the conversation 'back to square one,' so to speak. Progressivity may be achieved not only sequentially but also topically. Harry initiates a topic that names a class of things and for that topic to be developed, items from that class would be appropriate responses that would progress the conversation. But Russell produces the minimal response and topical progressivity is noticeably absent.

\section{Transforming a Story-invitation into a Question}

In naming a topic to be addressed and in providing the collection in terms of which such topical talk may progress, Harry can also be understood to be inviting his son to tell a story about the day's athletic activities but Russell declines to take up this story-invitation. In other words, Harry is asking for a report and Russell is declining to give him one. The preferred response is a story or a 'proper report' which would have contained at least some category of activity that was done during athletics. In asking questions such as this, persons can be understood to be 'taking an interest' in their co-participant's activities, in this case what they did in athletics that day. Russell's declination is achieved via a transformation of Harry's conversational object. Harry's 'story-invitation' is transformed into a mere question in that Russell produces a technically correct response-he produces an answer-given that the vehicle for the story-invitation was a question. However, he declines to take up the action projected by the question. A preferred response to the question as a question has been produced, that is, an answer has been produced but a dispreferred response has been produced in response to the story invitation. The 'evidence' that Russell's response is a dispreferred one is provided in Harry's reaction.

\section{Analyzing the Categorial Transformations}

Russell's transformations of a collection into a category and a story-invitation into a question are analyzed by his parents in the turns following, firstly by his father (Harry) and then by his mother (Jen). This analysis is categorial. The first step, in this analysis (line 003-004) is that Russell's answer is category bound, that is, it is typical of his son, so much so that Harry declares that he 'should have put money on that then'. This formulation is then developed into a question (line 006-007) which contains a choice between two categories of action: between 'having a conversation' and being 'weird'. The choice subsumes both of Russell's transformations-of athletics from a collection to a category and of a story-invitation to a questionunder the activity-collection 'being weird'. Furthermore, this local collection of activities comprising 'being weird' are also activities which are disqualified from the collection of activities comprising a 'normal conversation'. 
Jen, on the other hand, analyzes Russell's talk as comprising another category of talk which belongs in a collection she refers to as 'being Kevinish' (line 010). The 'Kevin' she is referring to (and there are several other references to this made in the course of the meal) is a character from The Harry Enfield Television Programme (later called Harry Enfield and Chums) called 'Kevin the teenager' who plays the part of an uncooperative and wise-cracking 13 year old. ${ }^{5}$ Furthermore, where Harry's analysis is understandably serious in tone (and possibly threatening), Jen follows up her analysis with some laughter which can be heard to propose, if not some further laughter on the part of its recipient (cf. Jefferson 1978) then at least some 'lightening of the developing confrontational mood'.

\section{Transforming 'Athletics' into a Collection}

These analyses and conjectures aside, however, what is especially noticeable in this section of the extract is Jen's transformation of her son's transformation of athletics.

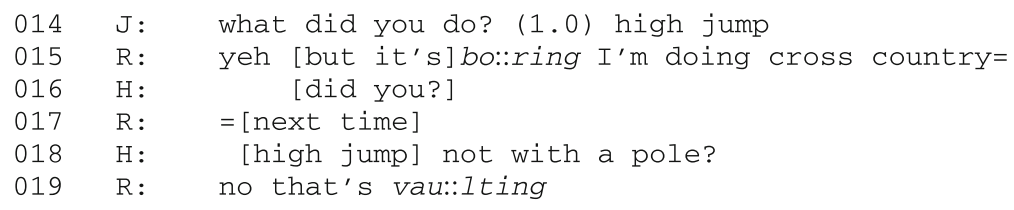

In asking Russell what he did and then in giving the answer herself 'high jump' Jen demonstrates the apparatus that Russell should use in taking up his father's story invitation. 'Athletics' is transformed back into a collection of activities comprising a number of different types of field sports, such as sprinting, cross country running, javelin throwing, long jump etc. High jump is also one of these categories and the one that Russell had been engaged in during his attendance at the athletics club that day. As can be seen from this extract Harry also joins in with a clarification request namely 'high jump not with a pole?' to which Russell responds 'no that's vaulting'.

Our interest here is in how Jo transforms the 'athletics' in Russell's answer from a single category into a category-collection. She does this by asking Russell, 'what did you do?' and she offers him a candidate answer, 'high jump'. In so doing, Jen's question can be heard to instruct Russell on the kind of answer that he should have provided in response to Harry's earlier story invitation. She 'educates' Russell in the preferred action to be taken in response to that storyinvitation by transforming 'athletics' back into a collection of activities and

\footnotetext{
5 Quoting Wikipedia on the Harry Enfield Television Programme, 'the term 'Kevin the teenager' (often shortened to simply a 'Kevin'), has entered British vernacular to describe any adolescent who is bad tempered or rebellious'.
} 
demonstrates to him how he should address it, namely by offering as a candidate answer one of the categories of activity included in the collection 'athletics'. He should not have treated 'athletics' as the category of activity that he engaged in, rather he should have treated athletics as a collection of categories of activity and should therefore have told a story which described the various categories of activity that he had done whilst at athletics club. It can be seen here, then, as in extract 1 above, that just as Jen transformed Russell's alternate category of potato into a collection of categories of potato with 'they're both normal,' so in this extract she again transforms a category into a collection.

Russell, in response to Jen's attempt to re-establish progressivity (Stivers 2006; Schegloff 2007a) with the question, 'What did you do?' followed by the candidate answer, 'High jump?,' not only confirms the candidate answer ('yeh') but then proceeds to categorize with a Kevin-style evaluation. Thus, he uses one of the standard lines associated with the character 'Kevin,' namely 'it's bo::ring'. Kevin uses this line in the show in response to a wide range of activities. The 'boring' is emphasized in that it is said loudly relative to the co-selected words and the 'bor' part of the word is elongated. Harry's 'did you' (line 016) overlaps with this quotation and his follow-up question, 'high jump not with a pole?' (line 018) overlaps with Russell's announcement that he 'will be doing cross country next time' (line 017). Nevertheless, both Russell and Harry are now assembling a collection-activities done in athletics today-and it can be seen that Russell is now speaking topically about athletics.

\section{Categorizing Russell's Conversational Style}

Just as conversational objects can be categorized and categorially transformed, so also may a speaker's conversational style or 'way of speaking' be categorized. Thus, persons may be said to be talking rudely, or harshly or aggressively for example. In this case, whilst it is difficult to know for sure whether Harry heard Russell speaking in the style of Kevin when he said "it's bo:::ring" (line 015) as an evaluation of high jump, what is clear is that when Russell repeats the style in his utterance 'no that's vau::lting' (line 019) in response to Harry's question, 'high jump not with a pole?' Harry's analysis is similar to that which he produced earlier (lines 006-007). Again, Russell is analysed as not having a 'normal conversation' with his father. That is to say, Harry's question 'high jump, not with a pole?' is a continuation of the conversation about categories of athletics and seeks confirmation or discomfirmation as to whether high jump involves a pole. Russell provides a preferred response, namely an answer to the question with a 'no' and an elaboration of the category of athletics that a pole is used in. However, Harry analyses the style in which this is done as another category of talking that belongs in the collection 'being weird' or 'not being normal' as opposed to having a 'normal' conversation. As he puts it: 


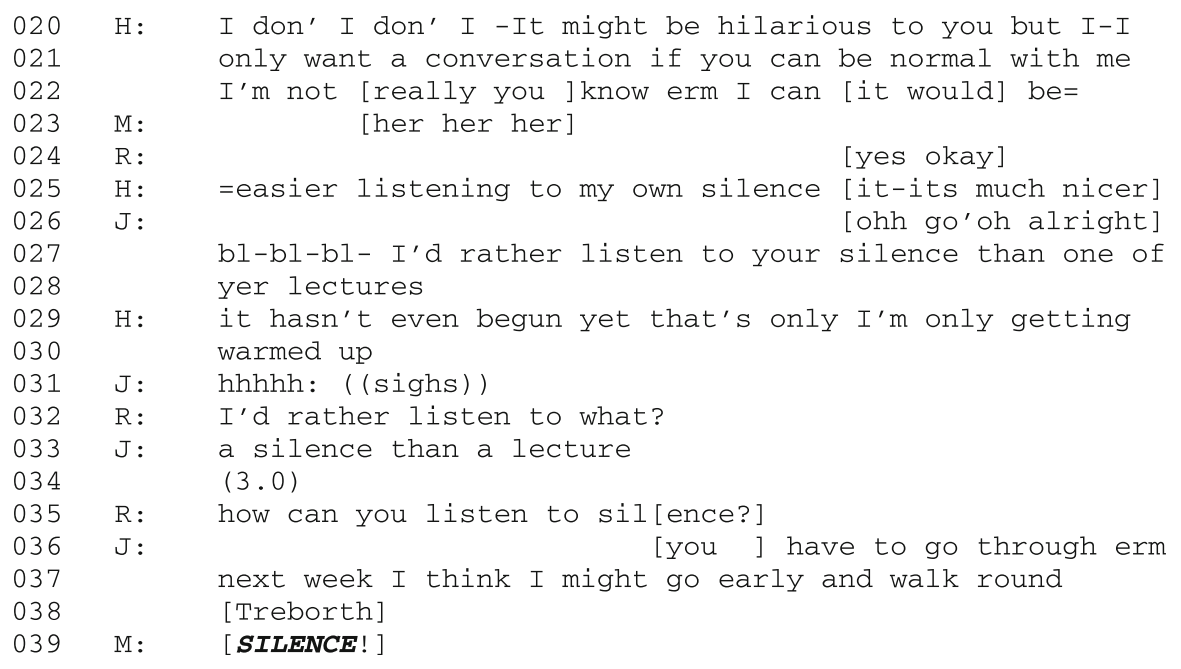

As the extract makes clear, unless Harry can have a 'normal conversation' with his son, he would rather listen to his own silence. As the extract above shows Jen supports this prospect by indicating that Harry's silence would be preferable to one of his lectures.

\section{Analyzing and Transforming Silence}

The exchange between Harry and Jen about Harry's silence presents Russell with an opportunity. He again appears to have an acute sense of the nuances of the meanings of words here. Jen's contrast between listening to a lecture and listening to silence is of course perfectly understandable if not semantically correct, since strictly speaking silence has no sound and cannot be heard. However, we 'know' what she means; such conversational looseness is something which conversationists tolerate as a matter of course. Russell, on the other hand, is alert to the playful opportunities afforded by this utterance and asks how in fact it is possible to 'listen to silence' (line 035). More thematically with respect to this discussion, Russell can be understood to be doing a category analysis of his parents' talk. In asking the question, 'how can you listen to silence?' he treats silence as one part of the contrast class: silence/noise, indicating by his question that he knows that listening is an activity that can be done with respect to noise but not with respect to silence. His reasoning appears to be that noise can be heard but its opposite, silence cannot, and therefore his parents' claim that they would prefer to listen to silence is a non sequiteur.

What is especially interesting, however, is that his sister, Maggie, takes up his analysis and the question Russell posed as a result of it, and in so doing she achieves a categorial transformation of silence. From being a category in the silence/contrast pair, silence is transformed into a constituent category of the collection 'words' (or 
'sounds,' since words, when spoken, have sounds). As such, silence also has a sound, and a noise can be made with this word-sound. With considerable wit, she pronounces loudly in answer to Russell's rhetorical question, 'silence' (line 039). Furthermore, she does not only say 'silence' loudly, she says it in a 'commanding' voice, possibly thereby accomplishing yet another transformation of 'silence' as a word-sound into 'silence' as an instruction. However, since her utterance is neither acknowledged nor analyzed by the other parties to the conversation it is difficult to be conclusive about this.

\section{Conclusion}

In this article we have been concerned with two main topics in MCA: (1) the occasionality or situated contingency of the 'machinery' or 'apparatus' used in members' MCA and (2) the phenomenon of categorial transformation which, we have sought to show, serves to illustrate the occasionality of MCA as well as comprising an interactional practice worthy of investigation in its own right. As we indicated earlier, previous work on categorial transformations of various kinds can be found in studies by Sharrock and Turner (1978), Jayyusi (1984), Eglin and Hester (2003) and in two 'classic' pieces by Sacks (1992a, Spring 1967, Lecture 13 and 1992b, Winter 1971, March 11). Our work has sought to build upon this literature by examining situated transformations of the apparatus of MCA itself. That is, we have shown how (1) erstwhile categories can be interactionally transformed into collections and vice versa, (2) categories of conversational object can be transformed into different categories of conversational object, (3) categories of object, including conversational objects (and ways of speaking) can be situationally subsumed under and assembled into various collections.

We also mentioned earlier that the emphasis on MCA as occasioned or situationally contingent phenomena exhibits an analytical focus on 'culture-inaction' as opposed to culture as a decontextualised resource for analyst and member alike. This latter notion finds expression in the potential for 'culturalism' which Sacks sought to avoid in his studies even as some of his more stipulative remarks could be understood as offering support for it (Hester and Eglin 1997a, b; Watson 1997). Indeed, as Schegloff (1992: xlii-xliv) remarks, it was for reasons of the 'potential promiscuity' of such culturalism that Sacks apparently 'abandoned' his concerns with membership categorization and sought to adhere to the kind of methodological rigor associated with sequential conversational analysis and its emphasis on the next turn proof procedure. In our view, it seems to be certainly the case that the language of MCA is largely absent from the lectures post 1968, but this does not necessarily indicate that Sacks had lost interest in categorial matters. As our discussion of his analysis of 'operative identities' and 'the 'old man' as an evolved natural object' indicates, the language of 'MCA' may be absent but an analytic concern with categorial issues remains. Furthermore, we would suggest that it exemplifies how a situationally contingent MCA can be undertaken, avoiding the pitfalls of culturalism and the attendant failure to treat seriously MCA as a members' phenomenon. Of course, Sacks did not explicitly respecify the 
'machineries' of MCA as local achievements but we would suggest his analyses of 'operative identities' and the like serve to demonstrate such achievements. We would not presume to conclude that our analysis approaches the brilliance of Sacks' work, but we would venture to suggest that a focus on categorial transformations can alert the analyst to the situational and occasioned character of MCA and can serve to encourage an adherence to the methodological commitments exemplified in Sacks' later work.

\section{References}

Baker, C. (2000). Locating culture in action: Membership categorization in text and talk. In A. Lee \& C. Poynton (Eds.), Culture and text: Discourse and methodology in social research and cultural studies. Allen and Unwin: Saint Leonard's, NSW.

Benson, D., \& Hughes, J. (1983). The Perspective of Ethnomethodology. London: Longman.

Buckner, H. T. (1970). Transformations of reality in the legal process. Social Research: An International Quarterly, 37(1), 88-101.

Coulter, J. (1982). Remarks on the conceptualization of social structure. Philosophy of the Social Sciences, 12(1), 33-46.

Coulter, J. (1991). Logic: Ethnomethodology and the logic of language. In G. Button (Ed.), Ethnomethodology and the human sciences. Cambridge: Cambridge University Press.

Eglin, P., \& Hester, S. (1992). Category, predicate and task: The pragmatics of practical action. Semiotica, 88, 243-268.

Eglin, P., \& Hester, S. (2003). The Montreal Massacre: A story of membership categorization analysis. Waterloo: Wilfrid Laurier University Press.

Francis, D., \& Hester, S. (2004). An invitation to ethnomethodology: Language, society and social interaction. London: Sage Publications.

Garfinkel, H. (1967). Studies in ethnomethodology. Englewood Cliffs: Prentice Hall.

Heritage, J. (1978). Aspects of the flexibilities of natural language use. Sociology, 12(1), 79-103.

Heritage, J. (1984). Garfinkel and ethnomethodology. Cambridge: Polity Press.

Hester, S. (1994). Les Categories en Contexte, Raisons Pratiques 5, $219-42$ (Special issue: B. Fradin, L. Quèrè, \& J. Widmer (Eds.), L'Enquete sur les Categories: de Durkheim a Sacks, Editions de l'Ecole des Hautes Etudes en Sciences Sociales, Paris.

Hester, S., \& Eglin, P. (1997a). Culture in action: Studies in membership categorization analysis. Lanham: International Institute for Ethnomethodology and Conversation Analysis and University Press of America.

Hester, S., \& Eglin, P. (1997b). The reflexive constitution of category, predicate and context in two settings. In S. Hester \& P. Eglin (Eds.), Culture in action: Studies in membership categorization analysis. Lanham: International Institute for Ethnomethodology and Conversation Analysis and University Press of America.

Jayyusi, L. (1984). Categorization and the moral order. London: Routledge and Kegan Paul.

Jefferson, G. (1978). Explanation of transcription notation. In J. Schenkein (Ed.), Studies in the organization of conversational interaction. New York: Academic Press.

Lee, J. R. E. (1984). Innocent victims and evil doers. Women's Studies International Forum, 7, 69-78.

McHoul, A., \& Watson, D. R. (1984). Two axes for the analysis of 'common sense' and 'formal' geographical knowledge in classroom talk. British Journal of Sociology of Education, 5, 281-302.

Payne, G. C. F. (1976). Making a lesson happen: An ethnomethodological analysis. In M. Hammersley \& P. Woods (Eds.), The process of schooling. London and Henley: Routledge and Kegan Paul and Open University Press.

Sacks, H. (1974). On the analyzability of stories by children. In R. Turner (Ed.), Ethnomethodology: Selected readings. Harmondsworth: Penguin Books.

Sacks, H. (1984). Notes on methodology. In J. M. Atkinson \& J. Heritage (Eds.), Structures of social action: Studies in conversation analysis. Cambridge: Cambridge University Press.

Sacks, H. (1992a). Lectures on conversation: Volume one. Oxford: Basil Blackwell. 
Sacks, H. (1992b). Lectures on conversation: Volume two. Oxford: Basil Blackwell.

Schegloff, E. (1992). Introduction, in H. Sacks (1992a). Lectures on Conversation: Volume one. Oxford: Basil Blackwell.

Schegloff, E. (1993). Reflections on talk and social structure. In D. Boden \& D. Zimmerman (Eds.), Talk and social structure: Studies in ethnomethodology and conversation analysis. Cambridge: Polity Press.

Schegloff, E. (2007a). Sequence organization in interaction: A primer in conversation analysis: Volume one. Cambridge: Cambridge University Press.

Schegloff, E. (2007b). A tutorial on membership categorization. Journal of Pragmatics, 39(3), 462-482.

Sharrock, W. W. (1974). On owning knowledge. In R. Turner (Ed.), Ethnomethodology: Selected readings. Harmondsworth: Penguin.

Sharrock, W. W., \& Turner, R. (1978). On a conversational environment for equivocality. In J. Schenkein (Ed.), Studies in the organization of conversational interaction. New York: Academic Press.

Stivers, T. (2006). A preference for progressivity in interaction. Language in Society, 35, 367-392.

Stivers, T., \& Hayashi, M. (2010). Transformative answers: One way to resist a question's constraints. Language in Society, 39, 1-25.

Watson, D. R. (1997). Some general reflections on 'categorization' and 'sequence' in the analysis of conversation. In S. Hester \& P. Eglin (Eds.), Culture in action: Studies in membership categorization analysis. Lanham, MD: International Institute for Ethnomethodology and Conversation Analysis and University Press of America. 